\title{
Tailored high-contrast attosecond electron pulses for coherent excitation and scattering
}

\author{
Sergey V. Yalunin ${ }^{\circ}, *$ Armin Feist, and Claus Ropers ${ }^{\dagger}$ \\ IV. Physical Institute, University of Göttingen, 37077 Göttingen, Germany \\ and Max Planck Institute for Biophysical Chemistry, 37077 Göttingen, Germany
}

(Received 19 March 2021; accepted 7 June 2021; published 10 August 2021)

\begin{abstract}
Temporally shaping the density of electron beams using light forms the basis for a wide range of established and emerging technologies, including free-electron lasers and attosecond electron microscopy. The modulation depth of compressed electron pulses is a key figure of merit limiting applications. In this work, we present an approach for generating background-free attosecond electron pulse trains by sequential inelastic electron-light scattering. Harnessing quantum interference in the fractional Talbot effect, we suppress unwanted background density in electron compression by several orders of magnitude. Our results will greatly enhance applications of coherent electron-light scattering, such as stimulated cathodoluminescence and streaking.
\end{abstract}

DOI: 10.1103/PhysRevResearch.3.L032036

Actively shaped free-electron beams offer the capability for enhanced sensing and microscopy [1-7], with numerous applications employing transverse shaping, incorporating tailored phase masks [8-13], quasistatic electromagnetic optical elements [14-17], or scattering at the ponderomotive potential [18-20]. In the time domain, inelastic electron-light scattering (IELS) [21-25] leads to correlated gain or loss of angular $[26,27]$ and linear momenta with energy [28-30]. Following dispersive propagation, inelastic interactions can lead to a reshaping of the electron density into a train of attosecond pulses [24,31-33]. These states have been experimentally prepared and characterized by quantum state tomography [34] or streaking [35-38].

Besides their direct use for attosecond streaking and spectroscopy, such density-modulated electron beams are proposed for a multitude of applications, e.g., to imprint an external phase onto cathodoluminescence (CL) [39-42], to induce a microscopic polarization in two-level systems (TLSs), or to coherently build up mode amplitudes or local polarizations using independent electrons [43-48], thus promising a merger of electron microscopy with coherent spectroscopy.

A key limitation for these efforts is the quality of compression and the amount of uncompressed background density $[34,36,37,46,49]$, sometimes described by the classical multielectron bunching factor for self-amplified spontaneous emission based free-electron lasers [50-53]. However, even for a pure single-electron state [54-56] focused in the quantum regime [34], limited coherence arises [40,42-44,47].

\footnotetext{
*yalunin@gwdg.de

†cropers@gwdg.de
}

Published by the American Physical Society under the terms of the Creative Commons Attribution 4.0 International license. Further distribution of this work must maintain attribution to the author(s) and the published article's title, journal citation, and DOI. Open access publication funded by the Max Planck Society.
In this Letter, we directly address this issue by devising an experimentally feasible scheme to prepare essentially background-free attosecond electron pulse trains, drastically enhancing the coherence in electron-light interactions. Introducing a single-color, multiplane phase shaping approach, we predict tailored electron states representing excellent approximations to pointlike classical currents within the optical cycle. These states are capable of producing CL with a near-unity degree of coherence, a maximized microscopic polarization of few-level systems, and the enhanced coherent buildup of mode and transition amplitudes using multiple independent electrons.

The wave-function shaping scheme is depicted in Fig. 1. It is based on sequential temporal phase plates using IELS to modulate the electron momentum, each followed by a drift stage to transfer this momentum modulation into a train of attosecond density pulses [34-36]. As shown below, tailored precompression removes most of the electron density background and greatly enhances the coherence properties of a variety of excitations and scattering processes in the sample plane (Fig. 1, bottom).

The Letter is organized as follows. First, we briefly present a quantum mechanical approach to temporal phase plates and its classical limit. Second, the generation of nearly background-free attosecond electron pulses using sequential interactions is described. Finally, we discuss the consequences and features of using such electron states in selected applications, including streaking, CL, and the excitation of two-level systems.

Multislice method for electron propagation. There are various approaches to theoretically describe inelastic electronlight scattering and propagation [21-24]. Here, we employ a multislice approach [57], in which the forward propagation of electrons along the $z$ axis is described by the effective Schrödinger equation derived in the Supplemental Material [58]:

$$
i \hbar v \frac{\partial}{\partial z} \Psi(\mathbf{r}, t)=\left[H_{I}(\mathbf{r}, t)+D-\mathcal{E}\right] \Psi(\mathbf{r}, t),
$$


where $v$ is the mean electron velocity, $\mathcal{E}=i \hbar \partial_{t}$ the translation operator, and $H_{I}(\mathbf{r}, t) \approx-e v A_{z}(\mathbf{r}, t)$ the time-dependent scattering potential describing the interaction with light (see Supplemental Material [58]). The dispersion operator $D$ is given by

$$
D=\frac{\mathcal{E}^{2}}{2 \gamma^{3} m v^{2}}-\frac{\hbar^{2}}{2 \gamma m} \nabla_{\perp}^{2},
$$

where $\gamma=1 / \sqrt{1-v^{2} / c^{2}}$ is the Lorentz factor, $m$ the electron's rest mass, and $\nabla_{\perp}^{2}$ the Laplacian operator of the transverse coordinates. The Fresnel operator $U(h)=$ $\exp (-i D h / \hbar v)$ describes the propagation of the electron state from $z$ to $z+h$ without interaction. In the presence of an electromagnetic field, the solution of Eq. (1) can be obtained with the split-operator technique [59], using the Fresnel operator, the propagator for $D=0[23,30]$, and one of the higherorder decomposition schemes [60,61]. Since we are mainly interested in temporal or longitudinal focusing, for simplicity, we assume that the interaction is independent of $x, y$, separating the wave function into its temporal $\psi(z, t-z / v)$ and transverse parts, where the latter is known analytically for cylindrical beams $[10,62]$. The advancement of the temporal part from $z$ to $z+h$ is obtained by the second-order expression,

$$
\psi(z+h, t)=U(h / 2) e^{i \Phi(z, t)} U(h / 2) \psi(z, t),
$$

where the phase function is given by

$$
\Phi(z, t)=-\frac{1}{\hbar v} \int_{z}^{z+h} H_{I}(z, t+z / v) d z .
$$

This procedure is efficiently implemented with a fast Fourier transform algorithm. It is unitary and hence preserves the probability current $j=v|\psi(z, t)|^{2}$ integrated over time [63]. It follows from Eq. (3) that a scattering potential confined to an interval $(z, z+h)$ can be regarded as a thin inelastic or temporal phase plate located at $z+h / 2$, by analogy with elastic phase plates [20,64]. Thus, the forward propagation and temporal aberrations can be reduced to a phase function $\Phi(t)$ describing the temporal phase plate.

Classical limit. We first outline the classical picture of temporal aberrations $(\hbar \rightarrow 0)$, where $\hbar \Phi(t)$ has the meaning of a classical action. Its time derivative defines the change of the electron's energy and velocity,

$$
\Delta v(t)=-\frac{\hbar \Phi^{\prime}(t)}{\gamma^{3} m v} .
$$

Suppose that electrons uniformly distributed in time traverse a temporal phase plate at $z=0$ and gain a velocity change (5). The change of the probability density with increasing $z$ is determined by the trajectories,

$$
t(z)=t-\Delta v(t) z / v^{2} .
$$

The attracting fixed points in this map, i.e., the zeros of $\Delta v\left(t_{0}\right)$ with the time derivative $\Delta v^{\prime}\left(t_{0}\right)>0$, correspond to paraxial temporal foci. Paths concentrate and form a caustic near such points, resulting in narrow peaks in the density [49]. For a time-harmonic phase $\Phi(t)=2 g \cos (\omega t)$ characterized by an effective interaction strength $g$ (see an expression in the Supplemental Material [58]) and a frequency $\omega$, the attracting

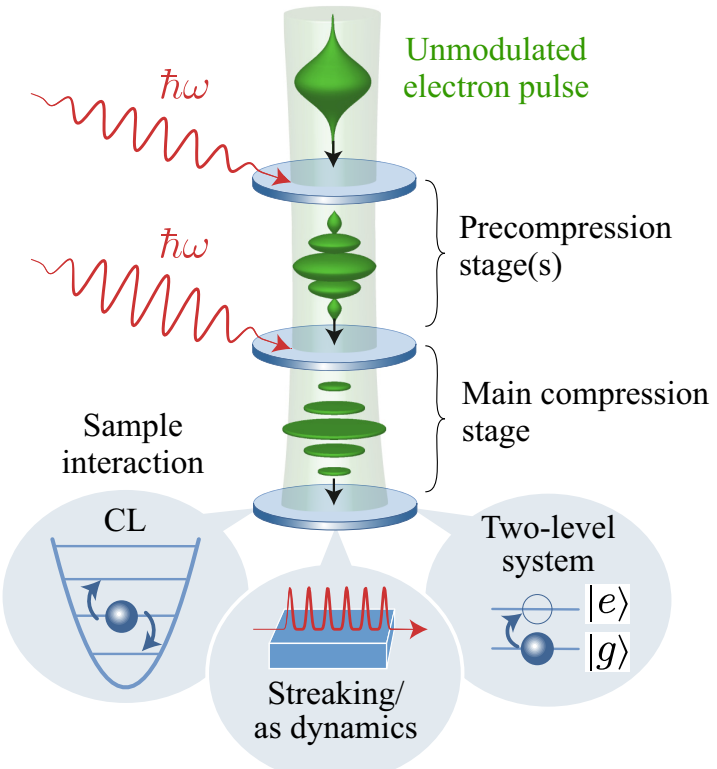

FIG. 1. Temporal electron pulse shaping and its prospective applications in attosecond physics, including streaking and the interaction with nanostructures and quantum systems.

points are given by $t_{0}=n T, n \in \mathbb{Z}$, with $T=2 \pi / \omega$ being the optical period. The paraxial focus lies at the distance $l_{f}$ from the phase plate, with

$$
l_{f}=\frac{v^{2}}{\Delta v^{\prime}\left(t_{0}\right)}=\frac{l_{T}}{8 \pi g}, \quad l_{T}=\frac{4 \pi m \gamma^{3} v^{3}}{\hbar \omega^{2}},
$$

where $l_{T}$ is the Talbot distance [65], which amounts to $200 \mathrm{~mm}$ for $120-\mathrm{keV}$ electrons and $800-\mathrm{nm}$ light.

Temporal focusing by monochromatic light is imperfect since paraxial trajectories do not converge into one point in the space-time diagram in Fig. 2(a), affecting the electron pulse duration. Another type of temporal aberration stems from repelling fixed points, at which half of the electrons are steered to a nearly homogeneous background in density [Fig. 2(a)]. The latter does not improve with increasing $g$ because it depends only on the product $g z$ [see Eq. (6)], and also phase-squeezed light does not reduce the background [65]. In principle, both types of temporal aberrations could be eliminated using multiple harmonics to approach a parabolic phase modulation of the form $\Phi(t)=-g \omega^{2} s^{2}(t)$, where $s(t)=(t+T / 2 \bmod T)-T / 2$ is the periodic sawlike function with zeros $n T, n \in \mathbb{Z}$. Generalized electron beam shaping using multiple harmonics has recently been theoretically considered [46], and two-color phase modulation experiments have been performed in the context of attosecond focusing and quantum state reconstruction [34]. However, superimposing an even larger number of harmonics with controlled amplitude and phase may render this approach rather impractical for attosecond focusing. Instead, as we show in the following, sequential monochromatic interactions in separate planes represent an even more powerful and experimentally tractable scheme to address temporal aberrations.

Eliminating focusing aberrations by sequential scattering. As described above, the aberrations in temporal focusing are most severe for those parts of the probability density 


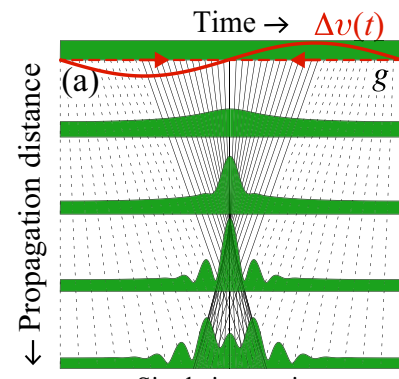

Single interaction
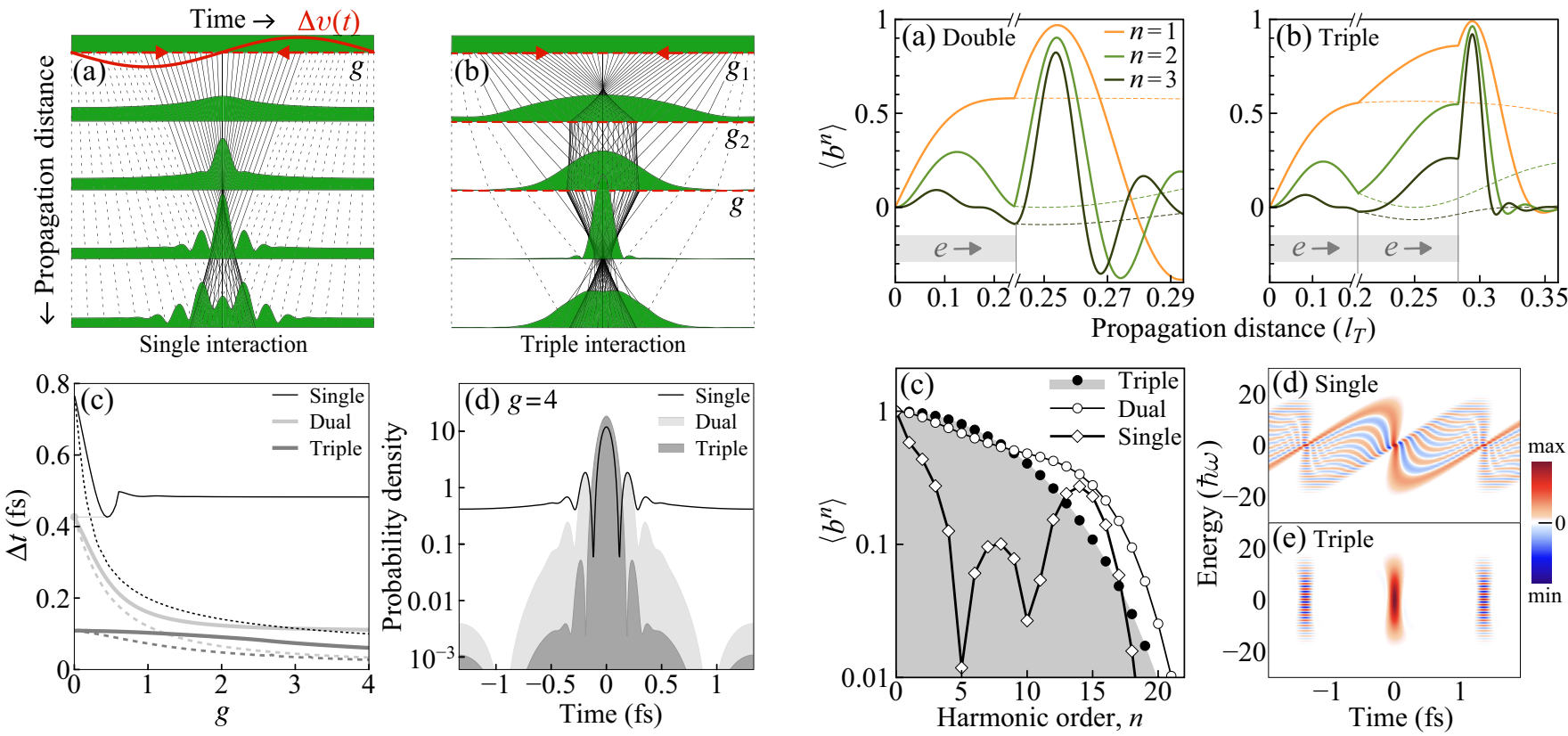

FIG. 3. (a),(b) Evolution of $\left\langle b^{n}\right\rangle$ (solid lines) after dual $\left(g_{1}=0.44\right)$ and triple $\left(g_{1}=0.39, g_{2}=0.52\right)$ interactions with the compression strength $g=4$. Dashed lines: The evolution after the first interaction only. (c) Values of $\left\langle b^{n}\right\rangle$ obtained at the distance where $\langle b\rangle$ reaches a maximum. (d),(e) Wigner distributions in the focus, showing a localization of electrons in phase space.

rms duration for single, dual, and triple monochromatic interaction as a function of the main compression strength $g$ (and free variation of the precompression strengths). Dashed lines: Respective durations when the main compression is a parabolic phase modulation. (d) Semilogarithmic plot of the density in the rms focus, illustrating a great reduction in the background density using sequential focusing.

furthest from the center of the cycle. We show here that weak precompression stages of interaction strength less than unity can reshape the density for much more efficient focusing [cf. Fig. 2(b)]. Specifically, the fractional Talbot effect [66] offers a powerful way to suppress the density near the repelling points, without adding further phase aberrations. At one-fourth of the Talbot distance, the wave function around the attractive points is a superposition of the phase-modulated initial state $\psi(0, t) \approx \psi_{0}\left(t_{0}\right) \exp \left[2 i g_{1} \cos (\omega t)\right]$ and its replica shifted by half of the optical period $[67,68]$ :

$$
\psi\left(l_{T} / 4, t\right)=\frac{\exp (-i \pi / 4)}{\sqrt{2}}[\psi(0, t)+i \psi(0, t+T / 2)] .
$$

Importantly, due to destructive interference, the probability density

$$
\rho\left(l_{T} / 4, t\right) \approx\left|\psi_{0}\left(t_{0}\right)\right|^{2}\left\{1+\sin \left[4 g_{1} \cos (\omega t)\right]\right\}
$$

vanishes at the repelling points $t=t_{0} \pm T / 2$ when $g_{1}=\pi / 8$. While such a modulation itself does not provide much temporal compression, a second stronger temporal phase plate completes the focusing, but now with greatly reduced background density.

Depending on the specific application, several properties can be considered for assessing the quality of attosecond focusing. A good measure of background density is given by the root-mean-square (rms) duration $\Delta t(z)$,

$$
\Delta t(z)=\sqrt{\left\langle s^{2}\right\rangle}, \quad\left\langle s^{2}\right\rangle=\frac{1}{C} \int_{-\infty}^{\infty} s^{2}(t) \rho(z, t) d t,
$$

where $\rho(z, t)=|\psi(z, t)|^{2}$ is the probability density, $C$ its integral, and $s(t)$ the sawlike function. Figure 2(c) illustrates the significant improvement of attosecond focusing in terms of the rms duration upon adding one or two prefocusing stages. As a function of the main compression strength $g$, $\Delta t(z)$ is evaluated at the respective minima along $z$, for a single interaction, a parabolic phase profile, and the optimized dual and triple (two weak and one strong) interactions. It is evident that the single-interaction case is rather limited in terms of its achievable rms duration, being minimized near $g \approx 0.45$ at $\Delta t \approx 0.426 \mathrm{fs}$. By an effective suppression of background density [see Fig. 2(d)], the dual interaction substantially reduces $\Delta t$. Although the most substantial absolute improvement is already obtained for the case of a single precompression phase plate, adding additional interaction planes leads to a further optimization, as evident from the results for a total of three phase plates shown in Figs. 2(b)-2(d) (see, also, the Supplemental Material [58]). It is worth noting that the idealized parabolic phase modulation applied after the precompression stages leads to only minor additional improvements [see Fig. 2(c)], illustrating that the introduced prefocusing scheme indeed strongly reduces aberrations and provides rms durations very close to the theoretical minimum.

Applications of background-free pulses. We note that the background-free attosecond pulses produced by this scheme will be exceedingly useful in a number of recently proposed schemes for coherent interactions of free electrons with 
nanostructures and individual quantum systems $[69,70]$. This includes electron-mediated coherence transfer and the coherent buildup of excitations for multiple subsequent electrons $[39,40,42-48]$. In each of the underlying processes, the modulation amplitude of the electron density at the fundamental modulation frequency, or its harmonics, plays a major role. This is quantified by the expectation values or moments $\left\langle b^{n}\right\rangle$ of the ladder operator $b=\exp (i \omega t)$ [44,71]. Different terminologies relating to these expectation values have been used, including the degree of coherence [40], the coherence factor [65], or the bunching factor [39,52,53,72].

The moments arising from a single interaction, $\left\langle b^{n}\right\rangle=$ $J_{n}\left[4 g \sin \left(2 \pi n z / l_{T}\right)\right]$ (see, also, Ref. [44]), are limited by the maxima of the Bessel functions $J_{n}$. Our sequential interaction scheme results in a substantial and nearly simultaneous enhancement of $\left\langle b^{n}\right\rangle$ above these values, as shown in Figs. 3(a)-3(c). Here, we numerically optimized the first moment $\langle b\rangle$ by a variation of the precompression strengths $g_{1,2}$ and the respective distances $d_{1,2}$ between interaction planes, for a fixed value of $g=4$. For a dual interaction, the optimization yields a maximum value of $\langle b\rangle_{\max } \approx 0.97$ for $g_{1} \approx 0.44$ and $d_{1} \approx 0.24 l_{T}$, well above the theoretical maximum $\langle b\rangle_{\max }=\max \left[J_{1}(z)\right] \approx 0.58$ for a single interaction, discussed in Refs. [42,44]. Note that the optimum distance $d_{1}$ is indeed very close to the quarter Talbot distance. An additional precompression phase plate $g_{2}$ leads to a further enhancement [Fig. 3(b)], with values of $\langle b\rangle_{\max } \approx 0.99$ and 0.998 for $g=4$ and 20, respectively. The enhancements of the higher moments are equally striking, reaching a nearly Gaussian distribution spanning multiple orders for a triple interaction [Fig. 3(c)].

The background-free attosecond focusing has direct implications for electron-driven radiative emission in the form of CL [73-80]. Specifically, our results imply a degree of coherence of CL near unity [39-42]. Similarly, due to the pronounced localization in time [see Fig. 2(d)] and the large moments of $b$, the background-free attosecond electron pulses lead to an almost fully coherent excitation of TLSs with a transition energy $\Delta E=\hbar \omega$ [43-48]. The quantum state in this case can be readily described using the Bloch model of the density matrix [81]. For a TLS initially in the ground state, the excitation can be seen as a "rotation" of the Bloch vector $\mathbf{a}=-\hat{\mathbf{z}}$ around a unit vector $\mathbf{n}$ in the $x-y$ plane, $\mathbf{a}(\theta)=$ $\mathbf{a} \cos \theta+\langle b\rangle(\mathbf{n} \times \mathbf{a}) \sin \theta$, where $\theta$ is the rotation angle. This angle can be determined through the transition probability $P_{2}=\sin ^{2}(\theta / 2)$ and Eq. (11) in Ref. [43]. Unmodulated electrons, or electrons with small $\langle b\rangle$, lead to a loss of the partial information about the relative phase between the eigenstates of the TLS because of the large uncertainty in the transition time [82]. In contrast, modulated electrons with $\langle b\rangle \approx 1$ retain this information and lead to a purity of the final state of $\operatorname{Tr}\left(\rho^{2}\right)=1+\frac{1}{2}\left(\langle b\rangle^{2}-1\right) \sin ^{2} \theta$ near unity. Incidentally, the dipole moment of the final state is maximized. Moreover, due to minimized quantum entanglement between the interacting systems, subsequent excitations driven by independent electrons modulated by the same reference wave can coherently build up either the cathodoluminescence in a particular mode or the transition amplitude in a TLS [7,43,44], leading to transition probabilities scaling with $N^{2}$ for small $\theta$, where $N$

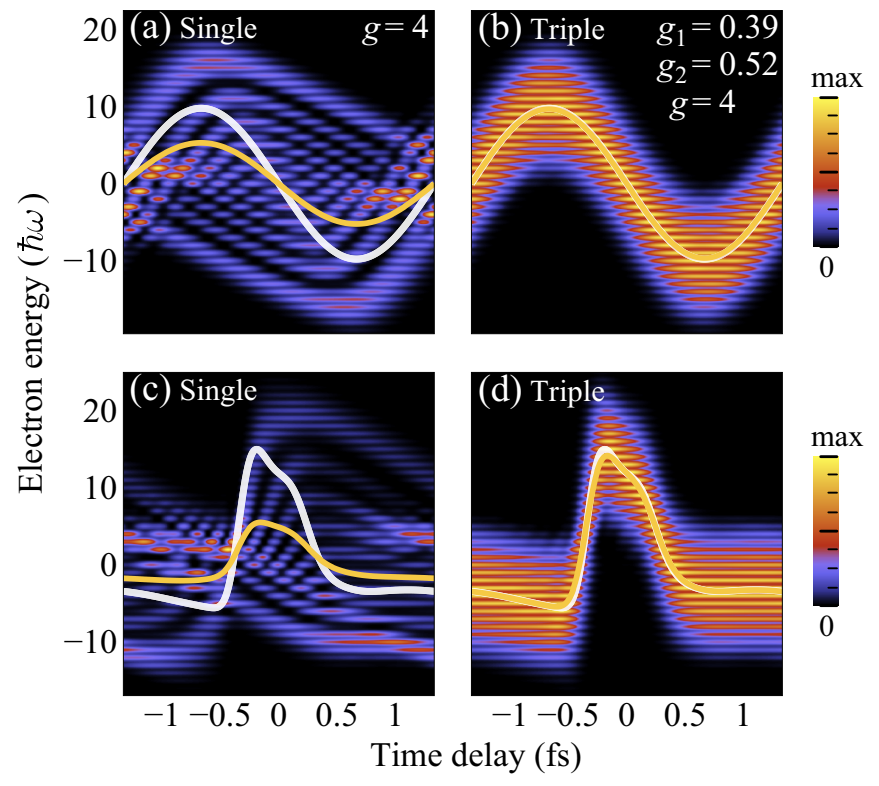

FIG. 4. Field-driven streaking spectrograms simulated with the electron pulses shown in Fig. 2(d). White lines: Temporal profile of the streak field $-\hbar \Phi^{\prime}(\tau)$, where $\Phi(\tau)$ is given by Eq. (4). Yellow lines: Calculated mean electron energy, $E(\tau)$.

is the number of electrons incident within the decoherence or dephasing time.

We note that the electron pulses produced here can be regarded as optimized approximations to pointlike, classical phase-space densities, as is clearly seen by their Wigner function shown in Fig. 3(e). This makes them ideally suited for temporal probing of periodic electromagnetic fields by means of free-electron energy or angular streaking [25,35-38]. In energy streaking, a time-periodic electric field $F(\tau)=\sum_{n} c_{n} e^{i n \omega \tau}$ or, more precisely, its spatial Fourier components in Eq. (4) are mapped onto the energy domain via the expression $E(\tau) \sim\langle F(t+\tau)\rangle=\sum_{n}\left\langle b^{n}\right\rangle c_{n} e^{i n \omega \tau}$, where $E(\tau)$ is the displacement of the mean energy of electrons, and $\tau$ the time delay between the electron pulse train and the streak field. Of course, the rather limited moments $\left\langle b^{n}\right\rangle$ resulting from single-field focusing [see Fig. 3(c)] strongly affect the performance of the streaking technique. Furthermore, as shown in Figs. 4(a) and 4(c), the presence of a background in electron pulses produced with the conventional scheme leads to pronounced spectral distortions. In contrast, the background-free electron pulses produced with our sequential focusing scheme lead to an almost perfect temporal representation of the streak field [see Figs. 4(b) and 4(d)].

Possible experimental implementations of our focusing scheme can be realized over a wide range of energies accessible in scanning and transmission electron microscopes. The main design parameter to consider is the Talbot distance [Eq. (7)], which scales with the cube of the electron velocity. Specifically, the precompression distance $l_{T} / 4$ at $800 \mathrm{~nm}$ optical wavelength reduces from $120 \mathrm{~mm}$ at $200 \mathrm{keV}$ to $8.6 \mathrm{~mm}$ at $40 \mathrm{keV}$ and $1 \mathrm{~mm}$ at $10 \mathrm{keV}$, dimensions for which technical solutions should readily be found. 
Conclusion. In summary, we have theoretically demonstrated how the concept of sequential scattering by light and the fractional Talbot effect can be applied to generate high-contrast attosecond electron pulses. We achieve an efficient correction of temporal aberrations, which leads to greatly enhanced coherence properties in electron-light scattering as compared to conventional temporal compression. Although the core of our generation scheme is a true quantum effect, harnessing destructive interference of the wave function, the resulting electron states exhibit an almost entirely positive Wigner function and greatly improved localization in phase space. Such states closely represent classical currents and produce practically fully coherent excitation and radiation at the modulation frequency.
Finally, we believe that similar schemes may also be applicable for free-electron lasers operating in the quantum regime [54-56].

Acknowledgments. We thank K. E. Priebe, Th. Rittmann, H. Lourenço-Martin, O. Kfir, V. Di Giulio, and F. J. García de Abajo for insightful discussions. This work was funded by the Deutsche Forschungsgemeinschaft (DFG, German Research Foundation) Grants No. 432680300/SFB 1456 (project C01) and No. 255652344/SPP 1840 (project 'Kohärente Wechselwirkungen starker optischer Nahfelder mit freien Elektronen'), the Gottfried Wilhelm Leibniz program, and the European Union's Horizon 2020 research and innovation program under Grant Agreement No. 101017720 (FET-Proactive EBEAM).
[1] V. Grillo, T. R. Harvey, F. Venturi, J. S. Pierce, R. Balboni, F. Bouchard, G. Carlo Gazzadi, S. Frabboni, A. H. Tavabi, Z.-A. Li, R. E. Dunin-Borkowski, R. W. Boyd, B. J. McMorran, and E. Karimi, Observation of nanoscale magnetic fields using twisted electron beams, Nat. Commun. 8, 689 (2017).

[2] A. Polman, M. Kociak, and F. J. García de Abajo, Electronbeam spectroscopy for nanophotonics, Nat. Mater. 18, 1158 (2019).

[3] H. Lourenço-Martins, D. Gérard, and M. Kociak, Optical polarization analogue in free electron beams, Nat. Phys. 17, 598 (2021).

[4] P. A. Midgley and R. E. Dunin-Borkowski, Electron tomography and holography in materials science, Nat. Mater. 8, 271 (2009).

[5] P. Kruit, R. Hobbs, C.-S. Kim, Y. Yang, V. Manfrinato, J. Hammer, S. Thomas, P. Weber, B. Klopfer, C. Kohstall, T. Juffmann, M. Kasevich, P. Hommelhoff, and K. Berggren, Designs for a quantum electron microscope, Ultramicroscopy 164, 31 (2016).

[6] K. Bliokh, I. Ivanov, G. Guzzinati, L. Clark, R. Van Boxem, A. Béché, R. Juchtmans, M. Alonso, P. Schattschneider, F. Nori, and J. Verbeeck, Theory and applications of free-electron vortex states, Phys. Rep. 690, 1 (2017).

[7] F. J. García de Abajo and A. Konečná, Optical Modulation of Electron Beams in Free Space, Phys. Rev. Lett. 126, 123901 (2021).

[8] M. Uchida and A. Tonomura, Generation of electron beams carrying orbital angular momentum, Nature (London) 464, 737 (2010).

[9] J. Verbeeck, H. Tian, and P. Schattschneider, Production and application of electron vortex beams, Nature (London) 467, 301 (2010).

[10] B. J. McMorran, A. Agrawal, I. M. Anderson, A. A. Herzing, H. J. Lezec, J. J. McClelland, and J. Unguris, Electron vortex beams with high quanta of orbital angular momentum, Science 331, 192 (2011).

[11] N. Voloch-Bloch, Y. Lereah, Y. Lilach, A. Gover, and A. Arie, Generation of electron Airy beams, Nature (London) 494, 331 (2013).

[12] V. Grillo, G. Carlo Gazzadi, E. Karimi, E. Mafakheri, R. W. Boyd, and S. Frabboni, Highly efficient electron vortex beams generated by nanofabricated phase holograms, Appl. Phys. Lett. 104, 043109 (2014).
[13] V. Grillo, A. H. Tavabi, F. Venturi, H. Larocque, R. Balboni, G. C. Gazzadi, S. Frabboni, P.-H. Lu, E. Mafakheri, F. Bouchard, R. E. Dunin-Borkowski, R. W. Boyd, M. P. J. Lavery, M. J. Padgett, and E. Karimi, Measuring the orbital angular momentum spectrum of an electron beam, Nat. Commun. 8 , 15536 (2017).

[14] B. J. McMorran, T. R. Harvey, and M. P. J. Lavery, Efficient sorting of free electron orbital angular momentum, New J. Phys. 19, 023053 (2017).

[15] T. R. Harvey, V. Grillo, and B. J. McMorran, Stern-Gerlach-like approach to electron orbital angular momentum measurement, Phys. Rev. A 95, 021801(R) (2017).

[16] J. Verbeeck, A. Béché, K. Müller-Caspary, G. Guzzinati, M. A. Luong, and M. Den Hertog, Demonstration of a $2 \times 2$ programmable phase plate for electrons, Ultramicroscopy $\mathbf{1 9 0 , 5 8}$ (2018).

[17] G. Pozzi, V. Grillo, P.-H. Lu, A. H. Tavabi, E. Karimi, and R. E. Dunin-Borkowski, Design of electrostatic phase elements for sorting the orbital angular momentum of electrons, Ultramicroscopy 208, 112861 (2020).

[18] P. L. Kapitza and P. A. M. Dirac, The reflection of electrons from standing light waves, Math. Proc. Cambridge Philos. Soc. 29, 297 (1933).

[19] D. L. Freimund, K. Aflatooni, and H. Batelaan, Observation of the Kapitza-Dirac effect, Nature (London) 413, 142 (2001).

[20] O. Schwartz, J. J. Axelrod, S. L. Campbell, C. Turnbaugh, R. M. Glaeser, and H. Müller, Laser phase plate for transmission electron microscopy, Nat. Methods 16, 1016 (2019).

[21] B. Barwick, D. J. Flannigan, and A. H. Zewail, Photoninduced near-field electron microscopy, Nature (London) 462 , 902 (2009).

[22] F. J. García de Abajo, A. Asenjo-Garcia, and M. Kociak, Multiphoton absorption and emission by interaction of swift electrons with evanescent light fields, Nano Lett. 10, 1859 (2010).

[23] S. T. Park, M. Lin, and A. H. Zewail, Photon-induced near-field electron microscopy (PINEM): Theoretical and experimental, New J. Phys. 12, 123028 (2010).

[24] A. Feist, K. E. Echternkamp, J. Schauss, S. V. Yalunin, S. Schäfer, and C. Ropers, Quantum coherent optical phase modulation in an ultrafast transmission electron microscope, Nature (London) 521, 200 (2015). 
[25] F. O. Kirchner, A. Gliserin, F. Krausz, and P. Baum, Laser streaking of free electrons at $25 \mathrm{keV}$, Nat. Photon. 8, 52 (2014).

[26] W. Cai, O. Reinhardt, I. Kaminer, and F. J. G. de Abajo, Efficient orbital angular momentum transfer between plasmons and free electrons, Phys. Rev. B 98, 045424 (2018).

[27] G. M. Vanacore, I. Madan, G. Berruto, K. Wang, E. Pomarico, R. J. Lamb, D. McGrouther, I. Kaminer, B. Barwick, F. J. García de Abajo, and F. Carbone, Attosecond coherent control of free-electron wave functions using semi-infinite light fields, Nat. Commun. 9, 2694 (2018).

[28] F. J. García de Abajo, B. Barwick, and F. Carbone, Electron diffraction by plasmon waves, Phys. Rev. B 94, 041404(R) (2016).

[29] G. M. Vanacore, G. Berruto, I. Madan, E. Pomarico, P. Biagioni, R. J. Lamb, D. McGrouther, O. Reinhardt, I. Kaminer, B. Barwick, H. Larocque, V. Grillo, E. Karimi, F. J. García de Abajo, and F. Carbone, Ultrafast generation and control of an electron vortex beam via chiral plasmonic near fields, Nat. Mater. 18, 573 (2019).

[30] A. Feist, S. V. Yalunin, S. Schäfer, and C. Ropers, High-purity free-electron momentum states prepared by three-dimensional optical phase modulation, Phys. Rev. Research 2, 043227 (2020).

[31] C. M. S. Sears, E. Colby, R. Ischebeck, C. McGuinness, J. Nelson, R. Noble, R. H. Siemann, J. Spencer, D. Walz, T. Plettner, and R. L. Byer, Production and characterization of attosecond electron bunch trains, Phys. Rev. Spec. Top. - Accel. Beams 11, 061301 (2008).

[32] S. A. Hilbert, C. Uiterwaal, B. Barwick, H. Batelaan, and A. H. Zewail, Temporal lenses for attosecond and femtosecond electron pulses, Proc. Natl. Acad. Sci. 106, 10558 (2009).

[33] P. Baum and A. H. Zewail, 4D attosecond imaging with free electrons: Diffraction methods and potential applications, Chem. Phys. 366, 2 (2009).

[34] K. E. Priebe, C. Rathje, S. V. Yalunin, T. Hohage, A. Feist, S. Schäfer, and C. Ropers, Attosecond electron pulse trains and quantum state reconstruction in ultrafast transmission electron microscopy, Nat. Photon. 11, 793 (2017).

[35] M. Kozák, N. Schönenberger, and P. Hommelhoff, Ponderomotive Generation and Detection of Attosecond Free-Electron Pulse Trains, Phys. Rev. Lett. 120, 103203 (2018).

[36] Y. Morimoto and P. Baum, Diffraction and microscopy with attosecond electron pulse trains, Nat. Phys. 14, 252 (2018).

[37] N. Schönenberger, A. Mittelbach, P. Yousefi, J. McNeur, U. Niedermayer, and P. Hommelhoff, Generation and Characterization of Attosecond Microbunched Electron Pulse Trains via Dielectric Laser Acceleration, Phys. Rev. Lett. 123, 264803 (2019).

[38] D. S. Black, U. Niedermayer, Y. Miao, Z. Zhao, O. Solgaard, R. L. Byer, and K. J. Leedle, Net Acceleration and Direct Measurement of Attosecond Electron Pulses in a Silicon Dielectric Laser Accelerator, Phys. Rev. Lett. 123, 264802 (2019).

[39] Y. Pan and A. Gover, Spontaneous and stimulated emissions of a preformed quantum free-electron wave function, Phys. Rev. A 99, 052107 (2019).

[40] O. Kfir, V. Di Giulio, F. J. G. de Abajo, and C. Ropers, Optical coherence transfer mediated by free electrons, Sci. Adv. 7, eabf6380 (2021).
[41] A. Karnieli, N. Rivera, A. Arie, and I. Kaminer, The coherence of light is fundamentally tied to the quantum coherence of the emitting particle, Sci. Adv. 7, eabf8096 (2021).

[42] V. Di Giulio, O. Kfir, C. Ropers, and F. J. García de Abajo, Modulation of cathodoluminescence emission by interference with external light, ACS Nano 15, 7290 (2021).

[43] A. Gover and A. Yariv, Free-Electron-Bound-Electron Resonant Interaction, Phys. Rev. Lett. 124, 064801 (2020).

[44] Z. Zhao, X.-Q. Sun, and S. Fan, Quantum Entanglement and Modulation Enhancement of Free-Electron-Bound-Electron Interaction, Phys. Rev. Lett. 126, 233402 (2021).

[45] D. Rätzel, D. Hartley, O. Schwartz, and P. Haslinger, Controlling quantum systems with modulated electron beams, Phys. Rev. Research 3, 023247 (2021).

[46] O. Reinhardt and I. Kaminer, Theory of shaping electron wavepackets with light, ACS Photon. 7, 2859 (2020).

[47] A. Gover, B. Zhang, D. Ran, R. Ianconescu, A. Friedman, J. Scheuer, and A. Yariv, Resonant interaction of modulationcorrelated quantum electron wavepackets with bound electron states, arXiv:2010.15756.

[48] Y. Morimoto, P. Hommelhoff, and L. B. Madsen, Coherent scattering of an optically modulated electron beam by atoms, Phys. Rev. A 103, 043110 (2021).

[49] P. Baum, Quantum dynamics of attosecond electron pulse compression, J. Appl. Phys. 122, 223105 (2017).

[50] C. Feng, H. Deng, D. Wang, and Z. Zhao, Phase-merging enhanced harmonic generation free-electron laser, New J. Phys. 16, 043021 (2014).

[51] C. J. Hirschmugl, M. Sagurton, and G. P. Williams, Multiparticle coherence calculations for synchrotron-radiation emission, Phys. Rev. A 44, 1316 (1991).

[52] A. Gover, R. Ianconescu, A. Friedman, C. Emma, N. Sudar, P. Musumeci, and C. Pellegrini, Superradiant and stimulated-superradiant emission of bunched electron beams, Rev. Mod. Phys. 91, 035003 (2019).

[53] X. Deng, A. Chao, J. Feikes, A. Hoehl, W. Huang, R. Klein, A Kruschinski, J. Li, A. Matveenko, Y. Petenev, M. Ries, C. Tang, and L. Yan, Experimental demonstration of the mechanism of steady-state microbunching, Nature (London) 590, 576 (2021).

[54] R. Bonifacio, N. Piovella, and G. Robb, The quantum free electron laser: A new source of coherent, short-wavelength radiation: The quantum free electron laser, Fortschr. Phys. 57, 1041 (2009).

[55] P. Kling, E. Giese, R. Endrich, P. Preiss, R. Sauerbrey, and W. P. Schleich, What defines the quantum regime of the free-electron laser?, New J. Phys. 17, 123019 (2015).

[56] C. M. Carmesin, P. Kling, E. Giese, R. Sauerbrey, and W. P. Schleich, Quantum and classical phase-space dynamics of a free-electron laser, Phys. Rev. Research 2, 023027 (2020).

[57] J. M. Cowley and A. F. Moodie, The scattering of electrons by atoms and crystals. I. A new theoretical approach, Acta Crystallogr. 10, 609 (1957).

[58] See Supplemental Material at http://link.aps.org/supplemental/ 10.1103/PhysRevResearch.3.L032036 for the derivation of Eq (1) and the simulated space-time evolution of the probability density after inelastic scattering.

[59] J. A. Fleck, J. R. Morris, and M. D. Feit, Time-dependent propagation of high energy laser beams through the atmosphere, Appl. Phys. 10, 129 (1976). 
[60] H. Yoshida, Construction of higher order symplectic integrators, Phys. Lett. A 150, 262 (1990).

[61] M. Suzuki, General theory of higher-order decomposition of exponential operators and symplectic integrators, Phys. Lett. A 165, 387 (1992).

[62] A. Lubk, Paraxial quantum mechanics, in Advances in Imaging and Electron Physics, Vol. 206 (Elsevier, New York, 2018), pp. $15-58$.

[63] L. Reimer and H. Kohl, Transmission Electron Microscopy, Vol. 36 (Springer, New York, 2008).

[64] A. Konečná and F. J. G. de Abajo, Electron Beam Aberration Correction Using Optical Near Fields, Phys. Rev. Lett. 125, 030801 (2020).

[65] V. Di Giulio and F. J. García de Abajo, Free-electron shaping using quantum light, Optica 7, 1820 (2020).

[66] M. V. Berry and S. Klein, Integer, fractional and fractal Talbot effects, J. Mod. Opt. 43, 2139 (1996)

[67] J. Guigay, On fresnel diffraction by one-dimensional periodic objects, with application to structure determination of phase objects, Opt. Acta: Intl. J. Opt. 18, 677 (1971).

[68] P. Cloetens, J. P. Guigay, C. De Martino, J. Baruchel, and M. Schlenker, Fractional Talbot imaging of phase gratings with hard x rays, Opt. Lett. 22, 1059 (1997).

[69] V. Di Giulio, M. Kociak, and F. J. G. de Abajo, Probing quantum optical excitations with fast electrons, Optica 6, 1524 (2019).

[70] A. Ben Hayun, O. Reinhardt, J. Nemirovsky, A. Karnieli, N. Rivera, and I. Kaminer, Shaping quantum photonic states using free electrons, Sci. Adv. 7, eabe4270 (2021).

[71] A. Feist, N. Bach, N. Rubiano da Silva, T. Danz, M. Möller, K. E. Priebe, T. Domröse, J. G. Gatzmann, S. Rost, J. Schauss, S. Strauch, R. Bormann, M. Sivis, S. Schäfer, and C. Ropers, Ultrafast transmission electron microscopy using a laser-driven field emitter: Femtosecond resolution with a high coherence electron beam, Ultramicroscopy 176, 63 (2017).

[72] C. B. Schroeder, C. Pellegrini, and P. Chen, Quantum effects in high-gain free-electron lasers, Phys. Rev. E 64, 056502 (2001).
[73] X. Bendaña, A. Polman, and F. J. García de Abajo, Singlephoton generation by electron beams, Nano Lett. 11, 5099 (2011).

[74] X. Lin, S. Easo, Y. Shen, H. Chen, B. Zhang, J. D. Joannopoulos, M. Soljačić, and I. Kaminer, Controlling Cherenkov angles with resonance transition radiation, Nat. Phys. 14, 816 (2018).

[75] S. Mignuzzi, M. Mota, T. Coenen, Y. Li, A. P. Mihai, P. K. Petrov, R. F. M. Oulton, S. A. Maier, and R. Sapienza, Energymomentum cathodoluminescence spectroscopy of dielectric nanostructures, ACS Photon. 5, 1381 (2018).

[76] N. Talebi, S. Meuret, S. Guo, M. Hentschel, A. Polman, H. Giessen, and P. A. van Aken, Merging transformation optics with electron-driven photon sources, Nat. Commun. 10, 599 (2019).

[77] N. J. Schilder, H. Agrawal, E. C. Garnett, and A. Polman, Phase-resolved surface plasmon scattering probed by cathodoluminescence holography, ACS Photon. 7, 1476 (2020).

[78] N. van Nielen, M. Hentschel, N. Schilder, H. Giessen, A. Polman, and N. Talebi, Electrons generate self-complementary broadband vortex light beams using chiral photon sieves, Nano Lett. 20, 5975 (2020).

[79] J. Christopher, M. Taleb, A. Maity, M. Hentschel, H. Giessen, and N. Talebi, Electron-driven photon sources for correlative electron-photon spectroscopy with electron microscopes, Nanophotonics 9, 4381 (2020).

[80] R. Remez, A. Karnieli, S. Trajtenberg-Mills, N. Shapira, I. Kaminer, Y. Lereah, and A. Arie, Observing the Quantum Wave Nature of Free Electrons through Spontaneous Emission, Phys. Rev. Lett. 123, 060401 (2019).

[81] M. O. Scully and M. S. Zubairy, Quantum Optics, 1st ed. (Cambridge University Press, Cambridge, 1997).

[82] F. J. García de Abajo and V. Di Giulio, Optical excitations with electron beams: Challenges and opportunities, ACS Photon. 8, 945 (2021). 